\title{
Domestic protection
}

Several neurodegenerative diseases have a genetic basis, but the precise way in which the mutant genotype is translated into the pathological phenotype remains unclear. One possibility is that the mutant protein becomes toxic for the cell, leading to its demise. This concept has guided research aimed at establishing whether endogenous proteins can protect neurons from degeneration, pointing to new molecular targets for therapeutic intervention. Two recent studies illustrate the potential of this idea.

Some forms of Parkinson's disease (PD) have been linked to mutations in $\alpha$-synuclein, and it has been proposed that the mutant protein alters the function of the ubiquitinproteasome system. Petrucelli et al. tested this proposal and found that overexpressing mutant $\alpha$-synuclein increased the sensitivity of cultured cells to proteasome inhibitors, at least in part by directly reducing proteasomal activity. Catecholaminergic neurons were particularly vulnerable, a finding that is consistent with the marked susceptibility of dopaminergic neurons in PD. Crucially, the overexpression of parkin, a ubiquitin ligase that has also been linked to PD, had a protective effect that depended on its ligase activity.
In the case of Huntington's disease, the production of huntingtin with an expanded polyglutamine tract leads to cell degeneration. By expressing polyglutamine-containing fragments of huntingtin in neurons of Caenorhabditis elegans, Faber et al. screened for genes that suppressed cell degeneration and identified polyQ enhancer-1 (pqe-1). Loss of pqe-1 function exacerbated neurodegeneration, whereas its overexpression had a protective effect. The PQE-1 protein has a domain rich in glutamine and proline residues that seems to be important for its protective action. Although no clear homologue of PQE-1 has been found in humans, it will be tempting to explore whether proteins with an equivalent domain can exert a similar protection.

$$
\text { Juan Carlos López }
$$

\section{(i) References and links} ORIGINAL RESEARCH PAPERS Petrucelli, L. et al. Parkin protects against the toxicity associated with mutant $\alpha$-synuclein: proteasome dysfunction selectively affects catecholaminergic neurons. Neuron 36, 1007-1019 (2002) | Faber, P. W. et al. Glutamine/proline-rich PQE-1 proteins protect Caenorhabditis elegans neurons from huntingtin polyglutamine neurotoxicity. Proc. Natt Acad. Sci. USA 99, 17131-17136 (2002)

\section{FURTHER READING McNaught, $K$. St $P$. et al.} Failure of the ubiquitin-proteasome system in Parkinson's disease. Nature Rev. Neurosci. 2, 589-594 (2001) | Gusella, J. F. \& MacDonald, M. E. Molecular genetics: unmasking polyglutamine triggers in neurodegenerative disease. Nature Rev. Neurosci. 1, 109-115 (2000)

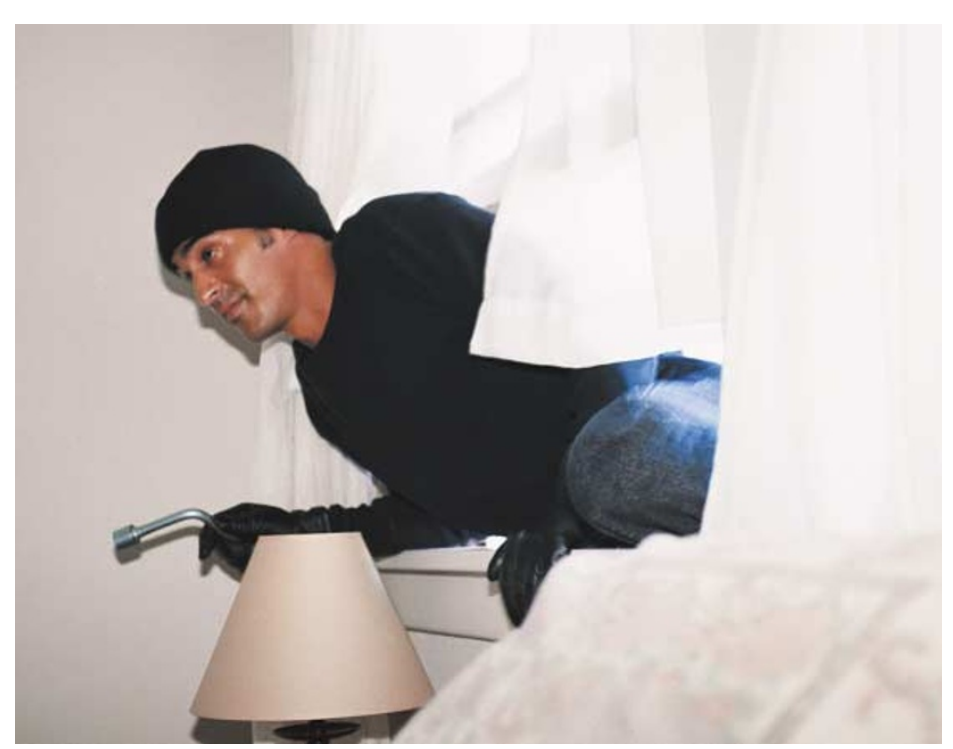

\section{IN BRIEF}

\section{CELL BIOLOGY OF THE NEURON}

Axonal transport of mitochondria to synapses depends on Milton, a novel Drosophila protein.

Stowers, R. S. et al. Neuron 36, 1063-1077 (2002)

To satisfy synaptic energy requirements, mitochondria must be able to traverse the often considerable distance that separates a terminal from its cell body. Here, Stowers et al. identify a Drosophila protein, Milton, which regulates mitochondrial movement. Without Milton, mitochondria fail to migrate from the cell body to axons and terminals - by contrast, transport of synaptic vesicles is unaffected. Coimmunoprecipitation indicated that Milton is associated with the kinesin heavy chain, and might therefore be required for the kinesin-mediated movement of mitochondria.

\section{ION CHANNELS}

Direct interaction with a nuclear protein and regulation of gene silencing by a variant of the $\mathrm{Ca}^{2+}$-channel $\beta_{4}$ subunit.

Hibino, H. et al. Proc. Natt Acad. Sci. USA 100, 307-312 (2003)

$\beta$-Subunits commonly regulate the functional properties of voltage-gated channels by a direct interaction with the poreforming $\alpha$-subunit. Here, the authors identify a splice variant of the $\beta_{4}$ subunit of $\mathrm{Ca}^{2+}$ channels $-\beta_{4 \mathrm{c}}$ - that, in addition to modifying channel behaviour, regulates gene expression. To carry out this novel function, $\beta_{4 c}$ directly binds to the transcriptional regulator $\mathrm{CHCB} 2 / \mathrm{HP} 1 \gamma$, markedly enhancing its gene-silencing activity.

\section{NEUROTRANSMITTERS}

Synthesis of serotonin by a second tryptophan hydroxylase isoform.

\section{Walther, D. J. et al. Science 299, 76 (2003)}

The rate-limiting enzyme in the synthesis of the neurotransmitter serotonin is tryptophan hydroxylase (TPH). Walther et al. show that mice without the gene that encodes TPH lack serotonin in the periphery but not in the brain, owing to the presence of a second isoform, Tph2, which is expressed only in the nervous system. The authors also found that $T p h 1$ is expressed almost exclusively in the periphery. $T p h 2$ is therefore more likely than $T p h 1$ to be important for brain function.

\section{NEUROPHYSIOLOGY}

Neuron-to-astrocyte signalling is central to the dynamic control of brain microcirculation.

Zonta, M. et al. Nature Neurosci. 6, 43-50 (2003)

Signalling from neurons to astrocytes could provide the key to the increase in cerebral blood flow that accompanies neuronal activity, according to Zonta et al. The authors showed that astrocytes increase their intracellular calcium concentration in response to the release of glutamate from neurons that synapse onto them. This in turn stimulates the release of vasoactive agents from the astrocytes, which cause arterioles to dilate. 